\title{
POWER ASYMMETRY IN COSMIC MICROWAVE BACKGROUND FLUCTUATIONS FROM FULL SKY TO SUB-DEGREE SCALES: IS THE UNIVERSE ISOTROPIC?
}

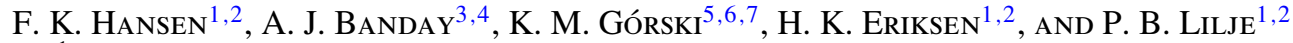 \\ ${ }^{1}$ Institute of Theoretical Astrophysics, University of Oslo, P.O. Box 1029 Blindern, N-0315 Oslo, Norway \\ ${ }^{2}$ Centre of Mathematics for Applications, University of Oslo, P.O. Box 1053 Blindern, N-0316 Oslo, Norway; frodekh@astro.uio.no, h.k.k.eriksen@astro.uio.no, \\ per.lilje@astro.uio.no \\ ${ }^{3}$ Max-Planck-Institut für Astrophysik, Karl Schwarzschild-Str. 1, Postfach 1317, D-85741 Garching bei München, Germany; banday@MPA-Garching.MPG.DE \\ ${ }^{4}$ Centre d'Etude Spatiale des Rayonnements, 9 av du Colonel Roche, BP 44346, 31028 Toulouse Cedex 4, France \\ 5 Jet Propulsion Laboratory, M/S 169/327, 4800 Oak Grove Drive, Pasadena, CA 91109, USA \\ ${ }^{6}$ Warsaw University Observatory, Aleje Ujazdowskie 4, 00-478 Warszawa, Poland \\ ${ }^{7}$ California Institute of Technology, Pasadena, CA 91125, USA; Krzysztof.M.Gorski@jpl.nasa.gov \\ Received 2008 December 19; accepted 2009 August 28; published 2009 October 2
}

\begin{abstract}
We repeat and extend the analysis of Eriksen et al. and Hansen et al., testing the isotropy of the cosmic microwave background fluctuations. We find that the hemispherical power asymmetry previously reported for the largest scales $\ell=2-40$ extends to much smaller scales. In fact, for the full multipole range $\ell=2-600$, significantly more power is found in the hemisphere centered at $\left(\theta=107^{\circ} \pm 10^{\circ}, \phi=226^{\circ} \pm 10^{\circ}\right)$ in galactic co-latitude and longitude than in the opposite hemisphere, consistent with the previously detected direction of asymmetry for $\ell=2-40$. We adopt a model selection test where the direction and amplitude of asymmetry, as well as the multipole range, are free parameters. A model with an asymmetric distribution of power for $\ell=2-600$ is found to be preferred over the isotropic model at the $0.4 \%$ significance level, taking into account the additional parameters required to describe it. A similar direction of asymmetry is found independently in all six subranges of 100 multipoles between $\ell=2-600$. None of our 9800 isotropic simulated maps show a similarly consistent direction of asymmetry over such a large multipole range. No known systematic effects or foregrounds are found to be able to explain the asymmetry.
\end{abstract}

Key words: cosmic microwave background - cosmology: observations - methods: data analysis - methods: statistical

\section{INTRODUCTION}

In the first public release of the Wilkinson Microwave Anisotropy Probe (WMAP) data (Bennett et al. 2003), we reported a significant asymmetry of the distribution of large-scale power on the sky (Eriksen et al. 2004b; Hansen et al. 2004b). This finding, as well as other statistical anomalies, was confirmed by several other authors using different methods (de Oliveira-Costa et al. 2004; Tegmark et al. 2003; Park 2004; Vielva et al. 2004; Eriksen et al. 2004a, 2004c; Land \& Magueijo 2005a, 2005b; Larson \& Wandelt 2004; McEwen et al. 2005; Hansen et al. 2004c, 2004a). Some of these have been confirmed again in the 3 and 5 year data (Hinshaw et al. 2007; Spergel et al. 2007; Cruz et al. 2007; McEwen et al. 2006; Eriksen et al. 2007; Pietrobon et al. 2008). Hansen et al. (2004b) found that the independent multipole ranges $\ell=2-19$ and $\ell=20-40$ were both particularly asymmetric, but with two different axes of asymmetry; the former being a galactic north-south asymmetry and the latter being an east-west asymmetry. Using the full range $\ell=2-40$, we found the highest significance of the asymmetry with the axis pointing in the direction of $\left(100^{\circ}, 237^{\circ}\right)$ in galactic co-latitude and longitude (this is the convention we will use for all sky positions in this paper).

The position of the non-Gaussian cold spot found by Vielva et al. (2004) is found to be positioned close to the center of the hemisphere with large fluctuation power. Furthermore, the cosmic microwave background (CMB) signal also demonstrated non-Gaussian statistical properties in the direction where the power spectrum amplitude was low (Park 2004; Eriksen et al. 2004c; Hansen et al. 2004c). Our own attempts to provide an explanation to the asymmetry invoked a class of homogeneous models that include anisotropic expansion (shear) and global rotation (vorticity) - the Bianchi-type VIIh models (Jaffe et al. 2005 , 2006a). Unfortunately, this fails as a physical explanation (Jaffe et al. 2006b; Bridges et al. 2007), since the best-fit parameter space is then inconsistent with a wide range of other evidences.

Recent theoretical developments (Ackerman et al. 2007; Erickcek et al. 2008; Gordon et al. 2005; Koivisto \& Mota 2008a, 2008b; Boehmer \& Mota 2008; Gumrukcuoglu et al. 2007a, 2007b) have led to proposals of new mechanisms for generating the imprint of a preferred direction in the CMB (see also Himmetoglu et al. 2009). Some of these models would lead to hemispherical asymmetry also for multipoles much larger than $\ell=40$ (angular scales smaller than $5^{\circ}$ ). A recent analysis (Groeneboom \& Eriksen 2009) of the Ackerman et al. model indicates a good fit to the WMAP data up to a multipole moment $\ell=400$. However, this model is not able to address the issue of the hemispherical power asymmetry.

In this paper, we re-investigate the nature of the power asymmetry using the recent 5 year data release from WMAP (Hinshaw et al. 2009), and specifically assess whether the signal manifests itself on smaller angular scales, $\ell \gg 40$. Instead of randomly searching for an asymmetry in arbitrary multipole ranges, we adopt a model selection procedure that searches for the best-fit asymmetry direction and multipole interval. An asymmetric model of the CMB needs more free parameters (i.e., the direction of the axis of asymmetry) than a simpler isotropic model. The model selection procedure tests whether an asymmetric model of the CMB is actually preferred by the data, taking into account the introduction of additional free parameters. We introduce a set of general asymmetric models with three to five additional parameters. These models are not based on any physical theory but are rather general 


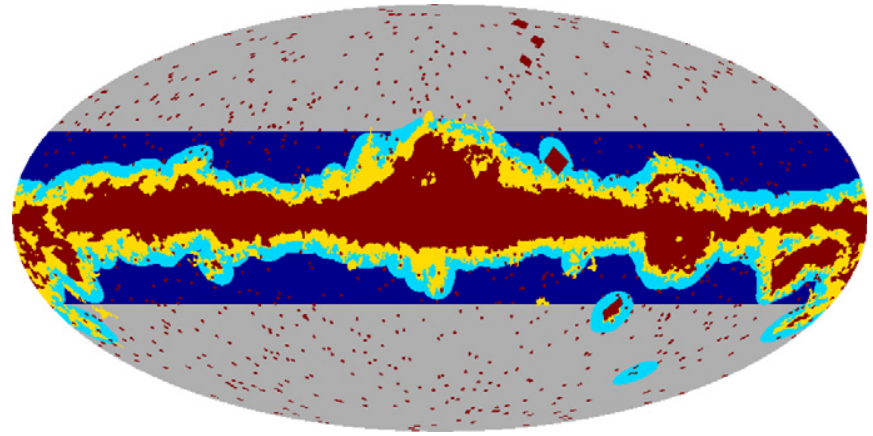

Figure 1. Different galactic cuts used in the paper: Kq85 (smallest), Kq75, $\mathrm{Kq} 75$ extended, and the largest $|b|>30^{\circ}$ cut. The point source mask, which is common for all masks, is also shown.

parameterizations of hemispherical asymmetry. We use these models in order to investigate how the asymmetry is distributed in harmonic space as well as on the sphere.

In Section 2, we describe the data and masks used in the analysis. The methods used to assess the asymmetry are outlined in Section 3. In Section 4, we show the results of the isotropy tests applied to the 5 year WMAP data, and in Section 5 we conclude.

\section{DATA}

The analysis in this paper was performed using the 5 year release of the WMAP data (publicly available at the Lambda Web site $\left.{ }^{8}\right)$ as well as a large ensemble of simulated maps of each of the channels Q $(41 \mathrm{GHz}), \mathrm{V}(61 \mathrm{GHz})$, and W $(94 \mathrm{GHz})$ (the result for each channel is obtained by taking the mean of all differencing assemblies - hereafter denoted as DAs-for each channel). All results in this paper are obtained using the foreground cleaned maps. From all maps, we have subtracted the best-fit mono and dipole. Some tests are also performed on maps from single DAs and single years of observation.

A series of galactic masks are used in the analysis:

1. KQ85: the WMAP KQ85 cut with a point source mask. Sky fraction used: $82 \%$;

2. KQ75: the WMAP KQ75 cut with a point source mask. Sky fraction used: $72 \%$

3. KQ75 ext.: the KQ75 cut extended with $5^{\circ}$ along the rim of the galaxy. Point source mask unchanged. Sky fraction used: $63 \%$;

4. $|b|>30$ (sometimes referred to as the $60^{\circ}$ cut): same as the extended KQ75 mask, but with an additional $60^{\circ}$ band cut on the galactic equator. Sky fraction used: $47 \%$;

5. KQ85N equals the $|b|>30$ cut in the northern galactic hemisphere and the KQ85 cut in the southern galactic hemisphere. Sky fraction used: $65 \%$;

6. KQ85S equals the $|b|>30$ cut in the southern galactic hemisphere and the KQ85 cut in the northern galactic hemisphere. Sky fraction used: $64 \%$.

In Figure 1, we show the different masks.

\section{METHODOLOGY: POWER SPECTRUM ESTIMATION ON HEMISPHERES}

\subsection{Hemisphere Spectra}

Previously (Eriksen et al. 2004b; Hansen et al. 2004b), we estimated the power spectrum on hemispheres centered on 164

\footnotetext{
8 http://lambda.gsfc.nasa.gov/
}

different positions on the sphere. In that earlier analysis, we applied the Gabor transform approach (Hansen et al. 2002; Hansen \& Górski 2003). In order to speed up the analysis without significant loss of precision, we will here apply the much faster MASTER algorithm (Hivon et al. 2002). This allows the analysis of many more positions on the sphere, as well as a large number of simulations for each WMAP year of observation and for each channel.

The power spectrum is estimated from the pseudo power spectrum by (Hivon et al. 2002)

$$
C_{\ell}=\sum_{\ell^{\prime}} K_{\ell \ell^{\prime}}^{-1}\left(\tilde{C}_{\ell^{\prime}}-N_{\ell^{\prime}}\right) \quad \tilde{C}_{\ell}=\sum_{m=-\ell}^{\ell} \frac{\tilde{a}_{\ell m}^{X} \tilde{a}_{\ell m}^{Y}}{2 \ell+1},
$$

where $\tilde{a}_{\ell m}^{X}$ is the spherical harmonic transform of channel $X$ with a given mask, $\tilde{C}_{\ell}$ is the corresponding pseudo power spectrum, and $N_{\ell}$ is the noise power spectrum. The coupling kernel $K_{\ell \ell^{\prime}}$ depends on the mask applied to the data as detailed by Hivon et al. (2002). In previous works, we only used the auto-spectra, i.e., $X=Y$. Here we will also use the cross power spectra $X \neq Y$ as an additional check. In the previous papers, we estimated the power spectrum on hemispheres centered on 164 different positions on the sphere. Here we use the positions of the 3072 pixel centers in a HEALPix ${ }^{9} N_{\text {side }}=16$ map.

Using the above approach, we obtain for each multipole bin $b$ an $N_{\text {side }}=16$ map, $M_{i}(b)$, where the value of each pixel $i$ corresponds to the $\ell(\ell+1) C_{\ell}$ power on a hemisphere centered on that pixel. In harmonic space, we bin the spectrum in 2 multipoles per bin, such that $\ell_{f}=2 b+2$ and $\ell_{l}=2 b+3$ are the first and last multipoles of bin $b$. We have also performed tests on more localized spectra, i.e., spectra estimated on disks of various sizes:

1. hemispheres (diameter $\left.180^{\circ}\right), 2$ multipoles per bin, $\ell_{f}=$ $2 b+2$ and $\ell_{l}=2 b+3$;

2. $90^{\circ}$ diameter disks, 4 multipoles per bin, $\ell_{f}=4 b+2$ and $\ell_{l}=4 b+5$

3. $45^{\circ}$ diameter disks, 16 multipoles per bin, $\ell_{f}=16 b+2$ and $\ell_{l}=16 b+17$

4. 22.5 diameter disks, 16 multipoles per bin, $\ell_{f}=16 b+2$ and $\ell_{l}=16 b+17$

In the previous papers, we tested for asymmetry in different multipole ranges using the set of opposite hemispheres which had the largest power spectrum ratio for each range. The problem with this approach is, however, that the maximum asymmetry axis, as well as the significance, is different for different multipole ranges. We made some attempts to determine whether the asymmetry continues to higher multipoles, and if so, what is the highest multipole where the asymmetry is present. This turned out to be difficult because of the instability of significances and directions as a function of the multipole range. Whether we have a significant asymmetry or not depends on which multipole range we choose to look at. Also the axis of asymmetry is slightly different for different multipole ranges.

We will now present an approach which first will solve the problem of choosing which multipole ranges to look at, and second will tell us to which degree a complicated asymmetric model is preferred by the data rather than the isotropic model. The approach is inspired by a similar idea proposed by Land \& Magueijo (2007). In order to understand the results from

\footnotetext{
9 http://healpix.jpl.nasa.gov/
} 
the model selection method, we will present a second, much simpler, asymmetry test, which is based on testing the alignment of the axes of asymmetry between independent multipole ranges.

\subsection{Model Selection Method}

We can look at the asymmetry in the following way: the asymmetry is the result of a dipole component in the maps, $M_{i}^{b}$, which is common for several multipole bins $b$. The dipole for a given multipole bin $b$ is given by

$$
a_{1 m}^{b}=\sum_{i} M_{i}^{b} Y_{1 m}^{i} .
$$

We thus propose the following asymmetric model,

$$
a_{1 m}^{b}=a_{1 m}^{b}(0)+A(b) a_{1 m}(\theta, \phi),
$$

where $a_{1 m}^{b}(0)$ is the random dipole expected in an isotropic model, and $a_{1 m}(\theta, \phi)$ is the common dipole component. The parameters $(\theta, \phi)$ are assumed to be independent of the bin. We will allow variations of the asymmetry amplitude $A$ with bin. To test this hypothesis, we will apply a simple $\chi^{2}$ fit,

$$
\chi^{2}=\mathbf{d}^{\dagger} \mathbf{C}^{-1} \mathbf{d},
$$

where the elements of the data vector $\mathbf{d}$ are given by $d_{m b}=$ $a_{1 m}^{b}(\mathrm{obs})-A(b) a_{1 m}(\theta, \phi)$ for all $m=[-1,1]$ and all bins $b$. Here $a_{1 m}^{b}(\mathrm{obs})$ is the dipole of the map $M_{i}^{b}$ for the data to be tested. The elements of the correlation matrix are simply $C_{m b, m^{\prime} b^{\prime}}=$ $\left\langle a_{1 m}^{b}\left(a_{1 m^{\prime}}^{b^{\prime}}\right)^{*}\right\rangle$. We will minimize this $\chi^{2}$ with respect to the parameters $(A(b), \theta, \phi)$. The resulting asymmetry direction is the best-fit common dipole component for all bins included in the analysis. The value of $\chi^{2}$ at the minimum will be compared to $\chi^{2}$ for the isotropic hypothesis,

$$
\chi_{0}^{2}=\mathbf{d}_{0}^{\dagger} \mathbf{C}^{-1} \mathbf{d}_{0},
$$

where the elements of $\mathbf{d}_{0}$ are given only by the observed dipole $d_{0, m b}=a_{1 m}^{b}(\mathrm{obs})$. The $\chi^{2}$ improvement $\Delta \chi^{2}=\chi_{0}^{2}-\chi^{2}$ due to the additional parameters will be calibrated with a set of isotropic Gaussian simulations. These simulations will give us the expected improvement $\Delta \chi^{2}$ from the additional parameters, with which the $\chi^{2}$ improvement in the data can be compared. If the improvement $\Delta \chi^{2}$ in the data turns out to be significantly better than in the simulations, this would indicate that the asymmetric model is preferred by the data.

We will test three different models for the asymmetry amplitude $A(b)$.

1. Constant amplitude. We will test (a) a three-parameter model $\left(A_{0}, \theta, \phi\right)$ with constant amplitude $A_{0}$ for a given set of multipole ranges; (b) a four-parameter model where the asymmetry is assumed to have the constant amplitude $A_{0}$ starting at $\ell=2$ until $\ell_{\max }$, where the latter is the fourth free parameter; (c) a five-parameter model where both the lowest and highest multipoles in the asymmetric multipole range, $\ell_{\min }$ and $\ell_{\max }$, are free parameters.

2. Linearly decreasing amplitude. In this four-parameter model, we will assume the asymmetry to be maximal at $\ell=2$ and decrease linearly according to $A=A_{0}(1-\alpha \ell)$, where $\alpha$ and $A_{0}$ are free parameters.

3. Gaussian multipole dependence. We will test a model where the asymmetry peaks at a certain multipole $\ell_{0}$ and falls off to both sides following a Gaussian, $A=A_{0} e^{-\left(\ell-\ell_{0}\right)^{2} /\left(2 \sigma^{2}\right)}$. We will test (a) a four-parameter model which is assumed to peak at $\ell_{0}=2$ with $\sigma$ as free parameter and a fiveparameter model where both $\ell_{0}$ and $\sigma$ are free parameters.

Before presenting the results, we will briefly describe the procedure that we use to obtain these results. The simulations are made with a maximum multipole of $L_{\max }$, so for model 1 above, the maximum and minimum multipoles (the free parameters $\ell_{\text {min }}$ and $\left.\ell_{\max }\right)$ will be sought within the multipoles available from the simulation in the range from $L_{\min }=2$ to $L_{\max }$. Since we will be looking for asymmetry extending over large multipole ranges, we will only look for multipole ranges with a minimum number of $\Delta \ell=\ell_{\max }-\ell_{\min }$ multipoles with a common dipole.

We made two sets of 1400 WMAP simulations (limited by the numbers of CPU hours and processors available) of a given band with a given mask. One set was used to construct the correlation matrix $\mathbf{C}$, while on the other set, as well as on the WMAP data, the $\chi^{2}$ minimization procedure was run in order to find the bestfit parameters. In order to obtain a converged covariance matrix with a limited number of simulations, we were forced to increase the size of the power spectrum bins to 20 multipoles (for the hemispheres and $90^{\circ}$ disks), for which the covariance matrix is well approximated as a diagonal matrix. For the smaller disks, we keep the original power spectrum bins of 16 multipoles.

The values for $\ell_{\min }, \ell_{\max }$, and $\theta$ which minimize $\chi^{2}$ were found using a three-dimensional grid, whereas the best-fit values of $\phi$ and $A_{0}$ were found analytically. The analytical expression for $\phi$ and $A_{0}$ is easily found by writing out $a_{1 m}$ as a function of $\theta$ and $\phi$ and setting the derivatives of $\chi^{2}$ with respect to $\phi$ and $A_{0}$ equal to zero. For each simulation, the difference $\Delta \chi^{2}=\chi_{0}^{2}-\chi^{2}$ between the minimum value of $\chi^{2}$ and $\chi_{0}^{2}$ for the isotropic hypothesis is recorded. This value shows the improvement in $\chi^{2}$ for this given simulation, when using an anisotropic model.

We then have an array of $\chi^{2}$ improvements for the given anisotropic model from all of the simulations, as well as for the data. We now check the $\chi^{2}$ improvement of the data with respect to the simulations. If we quote a significance of $1 \%$, it means that $1 \%$ of the simulations had a similar or larger improvement of $\chi^{2}$ using the anisotropic model. In the results, we will also list the best-fit multipole range $\left(\ell_{\min }, \ell_{\max }\right)$ as well as the amplitude $A$ and direction $(\theta, \phi)$ of the common dipole in the data.

In Figure 2, we show the preferred direction for 1400 simulated maps using various galactic cuts. The plot shows the density of best-fit directions as a function of galactic co-latitude. The KQ85 and KQ75 cuts show a uniform distribution of bestfit directions, whereas for the larger cuts there is a preference for the poles. Thus, we would expect that the preferred direction of asymmetry will be shifted away from the galactic plane for large sky cuts. Because of the large cut, power spectra estimated on hemispheres centered close to the poles will be similar in the polar area. This produces a dipolar structure with an axis pointing toward the poles.

\subsection{Test of Alignment of Multipole Ranges}

In Section 4 we will see, using the model selection method described above, that a model with a common dipole component in the range from $\ell=2$ to $\ell=600$ is preferred by the data with high significance. There should thus be a strong correlation between the distribution of power in different independent multipole ranges between $\ell=2$ and $\ell=600$. The purpose of 
Table 1

Significances (in \%) and Parameters $\left(\ell_{\max }, \theta, \phi, A_{0}\right)$ of the Best-fit Asymmetric Four-parameter Model

\begin{tabular}{lccccccc}
\hline \hline Channel & Mask & $\ell$-range & $\ell_{\max }$ & $\theta(\mathrm{deg})$ & $\phi(\mathrm{deg})$ & $p(\%)$ & $A_{0}\left(\mu K^{2} / 2 \pi\right)$ \\
\hline $\mathrm{Q}$ & $\mathrm{Kq} 85$ & {$[2,300]$} & 221 & $104 \pm 10$ & $226 \pm 10$ & 0.1 & $160 \pm 40$ \\
$\mathrm{~V}$ & $\mathrm{Kq} 85$ & {$[2,300]$} & $221(281)$ & $107 \pm 10$ & $226 \pm 10$ & $0.3(0.3)^{\mathrm{a}}$ & $150 \pm 40$ \\
$\mathrm{~W}$ & $\mathrm{Kq} 85$ & {$[2,300]$} & 221 & $103 \pm 9$ & $229 \pm 9$ & 0.1 & $170 \pm 40$ \\
$\mathrm{~V}$ & $\mathrm{Kq} 75$ & {$[2,300]$} & 281 & $112 \pm 14$ & $216 \pm 13$ & 3.1 & $120 \pm 40$ \\
$\mathrm{~V}$ & $\mathrm{Kq} 75$ ext. & {$[2,300]$} & 300 & $114 \pm 12$ & $202 \pm 13$ & 1.5 & $130 \pm 40$ \\
$\mathrm{~V}$ & $|b|>30$ & {$[2,300]$} & 300 & $131 \pm 18$ & $170 \pm 19$ & 17 & $100 \pm 40$ \\
\hline
\end{tabular}

Notes. Approximate Fisher matrix errors for $\theta, \phi$, and $A_{0}$ are also given. Please refer to the text for details about the asymmetric models and their parameters. The significances specify the percentage of simulated maps with a larger drop in $\chi^{2}$ for the asymmetric model (considering only models with $\Delta \ell>200$ ) than found in the WMAP data. The results are based on 1400 simulations.

a This result was obtained with 4200 simulations.

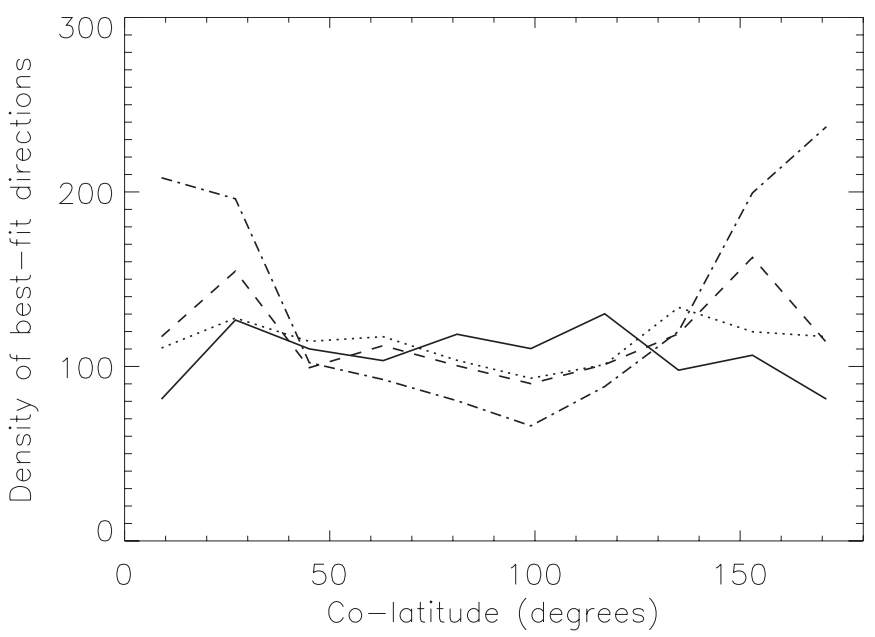

Figure 2. Preferred direction for 1400 isotropic simulations using the KQ85 cut (solid line), KQ75 cut (dotted line), extended KQ75 cut (dashed line), and the $60^{\circ}$ cut (dot-dashed line). Here, the amplitude, direction, and $\ell_{\max }$ are free parameters and may thus be different for each simulation.

the alignment test is to check if the asymmetry is distributed over the full multipole range and thus showing up as an alignment of the dipole of the power distribution of different independent multipole ranges.

The idea for the alignment test is simple. We construct the maps

$$
M_{i}(b 1, b 2)=\sum_{b=b 1}^{b 2} M_{i}(b),
$$

where we sum over (A) blocks of 20 multipoles and (B) blocks of 100 multipoles. Thus, for test A, we obtain a set of maps $M_{i}(2,21), M_{i}(22,41), M_{i}(42,61)$, etc., and for test B we obtain $M_{i}(2,101), M_{i}(102,201), M_{i}(202,301)$, etc. (because of different binning for $45^{\circ}$ and 22.5 tests, the ranges in test $\mathrm{B}$ will be as given in Table 3 ). The numbers of multipoles in the blocks, 20 and 100, have been chosen as a compromise between two factors; on the one hand we wanted bin sizes to be large enough to reduce the statistical noise, on the other hand we wanted them to be small enough to have a sufficient number of bins for the alignment check. We also required a set of large bins and a larger set of small bins, and therefore found 20 and 100 to be suitable bin sizes satisfying the two criteria.

For each map $M_{i}(b 1, b 2)$, the dipole is extracted (by a simple spherical harmonic transform on the map $\left.M_{i}(b 1, b 2)\right)$, and the direction of the dipole is stored in a vector $\vec{v}_{i}$ where $i$ is a multipole range $(b 1, b 2)$. In order to assess whether these directions for different multipole ranges are significantly more aligned in the WMAP data than in isotropic simulations, we define the mean angular distance $\bar{\theta}$ as

$$
\bar{\theta}=\sum_{i j} \arccos \left(\vec{v}_{i} \cdot \vec{v}_{j}\right),
$$

where the sum over $i$ and $j$ is over subranges $(b 1, b 2)$ up to the maximum multipole for the given case. We will in the following quantify the alignment of the power distribution in the WMAP data by specifying the number of simulations with a lower mean angular distance $\theta$ between the dipoles of the power distribution.

\section{RESULTS}

\subsection{Results with Model Selection}

As a first test of the model selection approach, we used the constant amplitude model with $\ell_{\min }$ and $\ell_{\max }$ fixed at the ranges $\ell=2-19$ and $\ell=2-41$, allowing only the amplitude and direction of asymmetry to vary. In this case, we found the bestfit asymmetry axis to be $\left(138^{\circ}, 220^{\circ}\right)$ and $\left(108^{\circ}, 227^{\circ}\right)$ being within $10^{\circ}-15^{\circ}$ of the asymmetry axis that Hansen et al. (2004b) found using the method described in Section 3.1. Note that due to the limited multipole range, we use power spectrum bins of two multipoles in this case, ignoring correlations between multipoles. In Figure 3, we show the distribution of $\chi^{2}$ improvements $\chi^{2}$ (isotropic) $-\chi^{2}\left(A_{0}, \phi, \theta\right)$, with the three parameters $\left(A_{0}, \phi, \theta\right)$ obtained with 1400 simulated isotropic maps. The vertical lines in the plots show $\Delta \chi^{2}$ for the data. For $\ell=2-41$, we see that only $0.4 \%$ of the simulations have a drop in $\chi^{2}$ similar to the drop seen in the data, showing that the anisotropic model is actually preferred by the data. For the multipole range $\ell=2-19$, however, $30 \%$ of the simulations show a similar drop in $\chi^{2}$, and this asymmetry is therefore not significant alone, taking into account the additional number of parameters required to describe it.

We now allow first $\ell_{\max }$ (fixing $\ell_{\min }=2$ ) and then later also $\ell_{\min }$ to be free parameters, i.e., we set the amplitude $A$ to a constant value $A_{0}$ in a multipole range $\left(\ell_{\min }, \ell_{\max }\right)$ and to zero for all other multipoles. Again we measured the drop in $\chi^{2}$ by the addition of four (fixing $\ell_{\min }=2$ and varying $\ell_{\max }$ ) and five parameters (varying both $\ell_{\min }$ and $\ell_{\max }$ ) for the simulations and compared to the data.

In Table 1, we show the results for larger scales. We extracted power spectra up to $L_{\max }=300$ from WMAP simulations of different channels with different galactic cuts. As we are looking for asymmetric models extending over a large range in multipole 

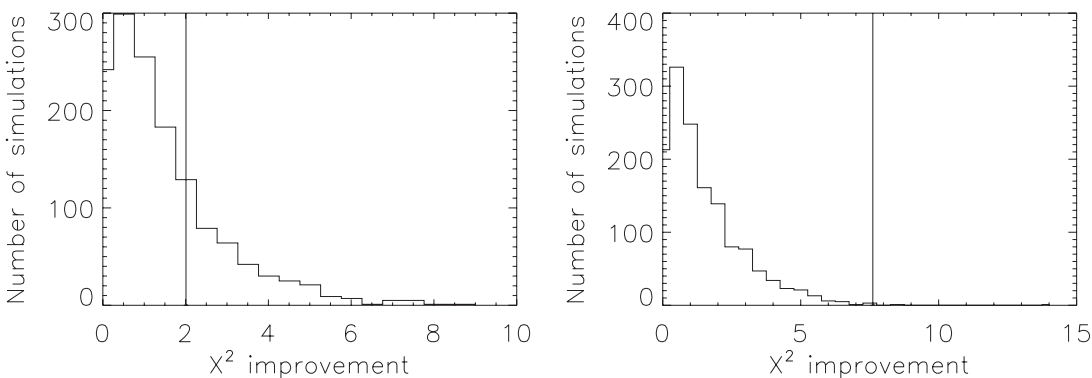

Figure 3. Histogram of the improvements in $\chi^{2}$ for a three-parameter model with $\left(\theta, \phi, A_{0}\right)$ as free parameters for 1400 simulated maps using the $W M A P V$-band parameters with the KQ85 galactic cut. The left plot is for a model with asymmetry in the range $\ell=2-19$, and the right plot is for a model with asymmetry in the range $\ell=2-41$. The vertical line is the improvement in $\chi^{2}$ for the 5 year WMAP data.

Table 2

Significances (in \%) and Parameters $\left(\ell_{\max }, \theta, \phi, A_{0}\right)$ of the Best-fit Asymmetric Four-parameter Model

\begin{tabular}{lccccccc}
\hline \hline Diameter & Mask & $\ell$-range & $\ell_{\max }$ & \multicolumn{1}{c}{$\theta(\mathrm{deg})$} & $\phi(\mathrm{deg})$ & $p(\%)$ & $A_{0}\left(\mu K^{2} / 2 \pi\right)$ \\
\hline $180^{\circ}$ & $\mathrm{Kq} 85$ & {$[2,500]$} & 481 & $102 \pm 12$ & $235 \pm 12$ & 2.3 & $70 \pm 20$ \\
$180^{\circ}$ & $\mathrm{Kq} 75$ & {$[2,500]$} & 481 & $104 \pm 16$ & $224 \pm 16$ & 14 & $50 \pm 20$ \\
$90^{\circ}$ & $\mathrm{Kq} 85$ & {$[2,800]$} & 601 & $105 \pm 11$ & $225 \pm 11$ & 2.6 & $130 \pm 30$ \\
$45^{\circ}$ & $\mathrm{Kq} 85$ & {$[2,800]$} & 591 & $102 \pm 9$ & $223 \pm 10$ & 0.6 & $170 \pm 40$ \\
22.5 & $\mathrm{Kq} 85$ & {$[2,800]$} & 591 & $107 \pm 11$ & $216 \pm 10$ & 0.4 & $\left(1.4^{\mathrm{a}} \pm 0.4\right) \times 10^{-4}$ \\
\hline
\end{tabular}

Notes. Approximate Fisher matrix errors for $\theta, \phi$, and $A_{0}$ are also given. Please refer to the text for details about the asymmetric models and their parameters. The significances specify the percentage of simulated maps with a larger drop in $\chi^{2}$ for the asymmetric model (considering only models with $\Delta \ell>400$ ) than found in the WMAP data. The results are based on 1400 simulations with the co-added $V+W$ channels.

${ }^{a}$ The power maps obtained from 22.5 disks are normalized (as described in the text), and the amplitude $A_{0}$ is therefore unitless in this case.

Table 3

Significances (in \%) and Parameters $\left(\ell_{\min }, \ell_{\max }, \theta, \phi, A_{0}\right)$ of the Best-fit Asymmetric Five-parameter Model

\begin{tabular}{lccccrrrr}
\hline \hline Diameter & Mask & $\ell$-range & $\ell_{\min }$ & $\ell_{\max }$ & $\theta(\mathrm{deg})$ & $\phi(\mathrm{deg})$ & $p(\%)$ & $A_{0}^{\mathrm{a}} \times 10^{-4}$ \\
\hline 22.5 & $\mathrm{Kq} 85$ & {$[2,95]$} & 2 & 63 & $110 \pm 15$ & $226 \pm 16$ & 8.4 & $0.38 \pm 0.14$ \\
22.5 & $\mathrm{Kq} 85$ & {$[96,191]$} & 96 & 191 & $100 \pm 16$ & $200 \pm 16$ & 19 & $0.27 \pm 0.10$ \\
22.5 & $\mathrm{kq} 85$ & {$[192,303]$} & 208 & 281 & $100 \pm 35$ & $238 \pm 34$ & 99 & $0.13 \pm 0.11$ \\
22.5 & $\mathrm{kq} 85$ & {$[304,399]$} & 352 & 399 & $83 \pm 24$ & $182 \pm 24$ & 77 & $0.23 \pm 0.13$ \\
22.5 & $\mathrm{kq} 85$ & {$[400,495]$} & 432 & 479 & $113 \pm 19$ & $224 \pm 19$ & 36 & $0.32 \pm 0.14$ \\
22.5 & $\mathrm{kq} 85$ & {$[496,591]$} & 496 & 591 & $112 \pm 20$ & $210 \pm 20$ & 48 & $0.21 \pm 0.10$ \\
22.5 & $\mathrm{kq} 85$ & {$[592,703]$} & 608 & 687 & $45 \pm 20$ & $111 \pm 24$ & 43 & $0.26 \pm 0.11$ \\
22.5 & $\mathrm{kq} 85$ & {$[704,799]$} & 736 & 799 & $35 \pm 26$ & $47 \pm 36$ & 63 & $0.25 \pm 0.13$ \\
\hline
\end{tabular}

Notes. Approximate Fisher matrix errors for $\theta, \phi$, and $A_{0}$ are also given. Please refer to the text for details about the asymmetric models and their parameters. The significances specify the percentage of simulated maps with a larger drop in $\chi^{2}$ for the asymmetric model than found in the WMAP data. The results are based on 1400 simulations for the co-added $V+W$ channels.

${ }^{a}$ The power maps obtained from 22.5 disks are normalized (as described in the text), and the amplitude $A_{0}$ is therefore unitless in this case.

space, we restrict our model search to models with at least 10 consecutive multipole bins (200 multipoles) with a common dipole component. The results for the four-parameter fits (the four free parameters are the constant amplitude $A_{0}$, the direction $(\theta, \phi)$ and the maximum multipole of asymmetry, $\left.\ell_{\max }\right)$ are presented in the table.

The table shows that within the multipole range $\ell=2-300$, there is a dipole component with common amplitude and direction for the multipole range $\ell=2-221$. For the KQ85 cut, only $0.1 \%-0.3 \%$ of the simulations show a similarly strong fit (similarly large $\chi^{2}$ improvement) for an asymmetric model. We see that the result is stable with the frequency channel. The significance is dropping with larger galactic cuts, but even with the extended KQ75 cut, only $1.5 \%$ of the simulations give a similarly strong fit to an asymmetric model. Even with the largest cut, the direction is remarkably consistent. Note, however, the expected shift away from the galactic plane, as discussed above. The dipole fitting procedure has thus revealed that the hemispherical power asymmetry extends to at least $\ell=221$.

The fact that the range $\ell=2-221$ is found to be the bestfit asymmetric range does not mean that the asymmetry cannot extend beyond $\ell=221$. Our model consists of an isotropic field, which in our analysis is considered noise, and an anisotropic dipole component, which is considered the signal. The isotropic "noise" component to the dipole will randomly change the 
direction and amplitude of each single power spectrum bin away from the asymmetric direction. By including larger multipole ranges, this noise component is reduced, and asymmetries extending over larger multipole ranges, even beyond $\ell=300$, can give a good fit.

From Table 1, we also see that the best-fit asymmetric multipole range is slightly larger for the larger galactic cuts. For KQ85, $\ell=2-221$ is the best-fit asymmetric range, whereas for KQ75 $\ell=2-281$ gives a better fit, and for larger cuts $\ell=2-300$ is preferred. As discussed above, the fact that a smaller range $\ell=2-221$ gives the best-fit for KQ85 does not mean that the larger range $\ell=2-281$ is a bad fit for this mask. In the table, we have included in the parenthesis the significance for $\ell=2-281$ for KQ85. Clearly $\ell=2-281$ is also significantly asymmetric for KQ85, but because of the random noise component, $\ell=2-221$ gives a slightly better fit.

Note that we have not included the results of the fiveparameter fits. The reason for this is that the five-parameter fits in all these cases show identical best-fit model parameters (that is, they show that the best-fit value for the fifth parameter $\ell_{\min }$ equals $\ell_{\min }=2$ for all cases) to the four-parameter fits.

To investigate properly the maximum multipole for asymmetry, we run a set of simulations with $L_{\max }=500$ and $L_{\max }=800$. In order to reduce the CPU time for the $L_{\max }=800$ case, we now use disks of $90^{\circ}, 45^{\circ}$, and 22.5 diameter. In this way, we also obtain more localized spectra. For the $45^{\circ}$ and 22.5 disks, we had to use multipole bins of 16 multipoles in the power spectrum estimation, and therefore also in the dipole fitting, instead of 20 used in the previous analysis. For the 22.5 disks, the variance of the power spectrum estimate close to the galactic plane was so large that the map $M_{i}^{b}$ needed to be normalized by its standard deviation (obtained from simulations) before a dipole fit could be performed. As a result, the values for the amplitude obtained in this case are different from the amplitudes obtained for other disk sizes.

The results are shown in Table 2. The hemisphere results with $L_{\max }=500$ show that the asymmetry extends to $\ell=481$ with a similar direction of asymmetry as for lower multipoles, but now with a lower significance $(p=2.3 \%)$. For the more localized spectra, however, the asymmetry is found highly significant ( $p=0.4 \%$ for the most localized spectra) for the range $\ell=2-600$ for all disk sizes, but no evidence is found for an asymmetry extending beyond $\ell=600$.

Finally, we will make a consistency check by performing the five-parameter dipole fit in individual subranges of 100 multipoles from $\ell=2$ to $\ell=800$ using 22.5 disks. Note that the size of these subranges is not exactly 100 , since each power spectrum bin has 16 multipoles. We show the results in Table 3. We see clearly that the best-fit direction in each subrange up to $\ell=600$ is consistent with the best-fit direction, $(\theta=107, \phi=216)$, for the full range $\ell=2-591$ for this disk size. The two bins above $\ell=600$, however, show a very different dipole direction. We see that the asymmetry can be seen as an alignment of the power distribution dipoles between multipoles from $\ell=2$ up to $\ell=600$. We will now study this alignment in more detail.

\subsection{Results with the Alignment Test}

Before looking at significances, we will illustrate the direction of the dipoles of individual 100 multipole blocks with some figures. In Figure 4, we show the distribution of power in the WMAP $V+W$ band data using hemispheres (the KQ85 cut was used in the power spectrum estimation). Each map shows the
Table 4

Calculated Mean Angle $\bar{\theta}$ Between the Dipole Directions for all Blocks of 20 Multipoles Within the Given Multipole Range

\begin{tabular}{lcccc}
\hline \hline \multicolumn{1}{c}{ Mask } & KQ85(V) & KQ75(V) & KQ75ext(V) & $|b|>30(\mathrm{~V})$ \\
\hline$\ell=2-300$ & 0.9 & 0.9 & 0 & 5.6 \\
$\ell=2-200$ & 1.8 & 5.5 & 0.8 & 13 \\
$\ell=2-100$ & 2.0 & 4.3 & 3.4 & 16 \\
$\ell=100-300$ & 9.0 & 7.4 & 1.8 & 35.5 \\
$\ell=200-300$ & 67.5 & 24.6 & 20 & 49.9 \\
\hline
\end{tabular}

Notes. The numbers given in the table are the percentages of simulated maps with a lower mean angle $\bar{\theta}$. The results are based on 1400 simulations. Zero entries mean that none of the simulated maps had a similarly low mean angle. Refer to the text for details of how $\bar{\theta}$ is calculated.

distribution of power, $M_{i}(b 1, b 2)$, for a given 100 multipole range. We have already seen by eyes that there is a clear dipolar distribution, and that the direction of the dipole is very similar in each case. In Figure 5, we show the position of the dipole for subranges of 100 multipoles. The color of the disk indicates the multipole range (see Table 3 for the exact ranges used). The results in this plot are taken from power spectrum estimates on disks with diameter 22.5 using the KQ85 galactic cut. We see that all the individual multipole ranges have dipoles pointing in a direction close to the best-fit dipole for the full range $\ell=2-600$, indicated by the white hexagon.

The WMAP team pointed out that $\ell=22$ is a strong negative outlier in the full sky power spectrum, and $\ell=40$ is a strong positive outlier. Hansen et al. (2004b) noted that these outliers in the full sky spectrum seem to be associated with the asymmetry; the high outlier at $\ell=40$ was associated with the high power in the hemisphere of maximum asymmetry, and the low outlier at $\ell=22$ was associated with the low power in the opposite hemisphere. In Figure 6, we show the distribution of power in these two bins, as well as for the first bin, $\ell=2-3$, and the bin $\ell=28-29$, which is a particularly asymmetric bin. The conclusions of Hansen et al. (2004b) still hold. One can see the same dipolar distribution of power, which is also seen in the maps of the six subranges within $\ell=2-600$ in Figure 4.

In Table 4, we show the results for the alignment test using blocks of 20 multipoles. We limit the maximum multipole to 300 , because noise is getting important after $\ell=300$, increasing the variance of the directions of blocks with only 20 multipoles. Later, for blocks of 100 multipoles, we will also consider higher multipoles, as the noise is reduced in each block when averaging over 100 instead of 20 multipoles. In the table, we have considered the alignment test for the $V$ band, using different galactic masks. The numbers indicate the percentage of simulations with a lower mean angle $\bar{\theta}$ between dipole directions. For $\ell=2-300$, there is a significant ( $\sim 1 \%$ level) alignment between dipole directions for the 20 multipole blocks. Note in particular that for the extended KQ75 cut, none of the 1400 simulations are as strongly aligned as the WMAP data. Thus, the asymmetry is strong even with a large galactic cut. We also see that there is a significant (2\%-4\% level) alignment when considering only the first five 20 multipole blocks on large scales, $\ell=2-100$. Considering only the blocks on small scales, $\ell=200-300$, excluding the first 100 multipoles, there is no significant alignment present, however.

In Table 5, we show the results from the alignment test of 100 multipole blocks, using more localized spectra. The $V+W$ map with the KQ85 galactic cut was used in this analysis. We clearly see the result of the strong alignment, which was already 
$1=2-101$

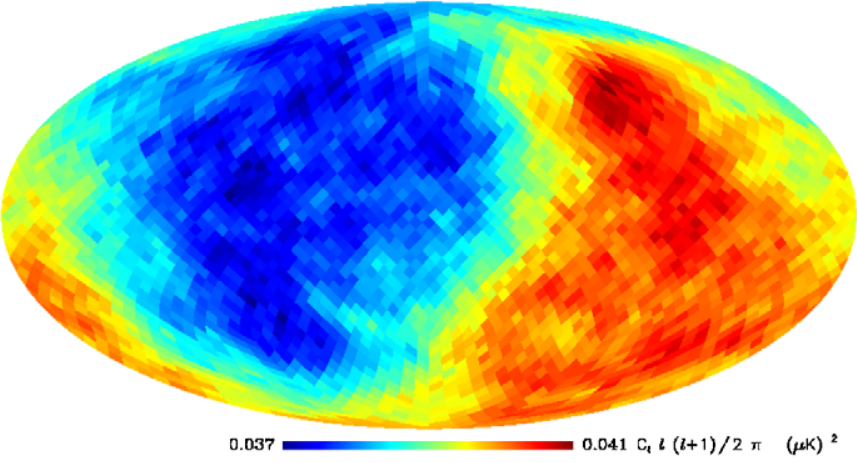

$1=202-301$

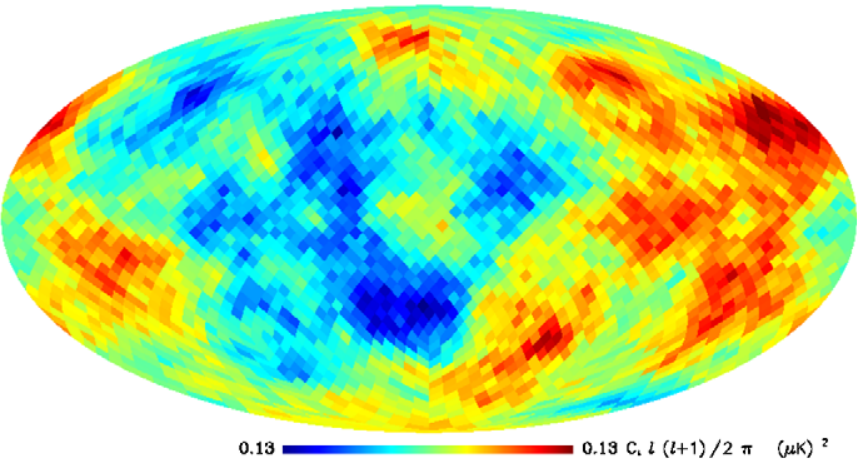

$1=402-501$

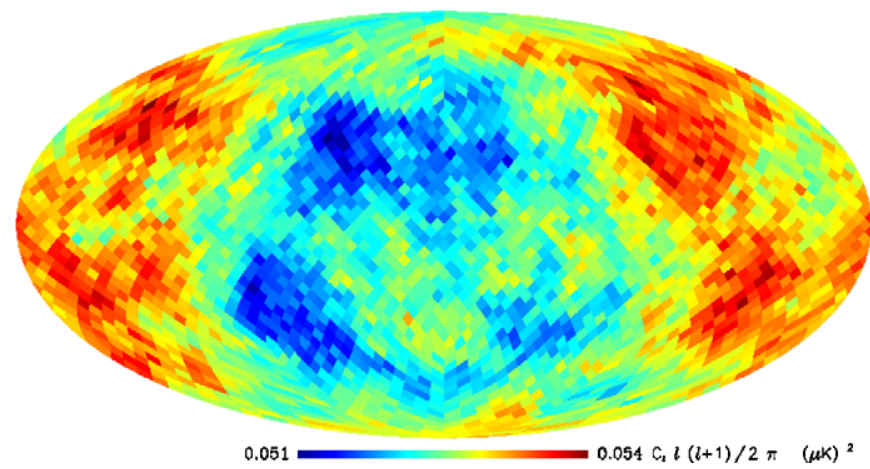

$1=102-201$

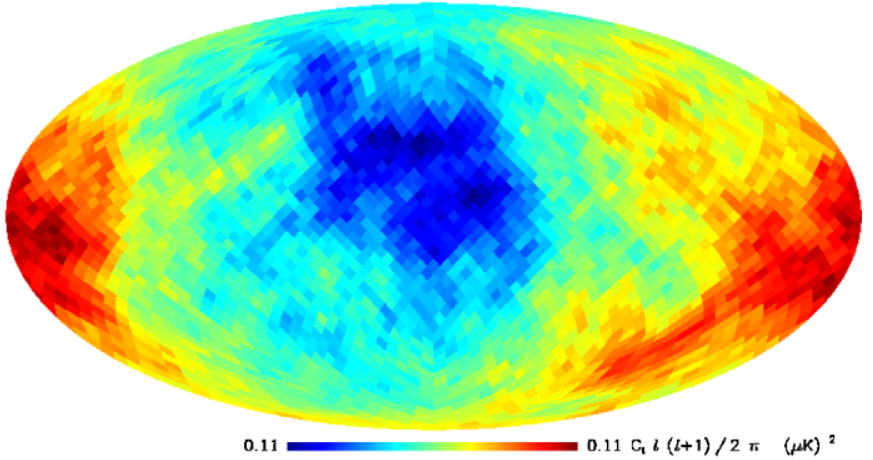

$1=302-401$

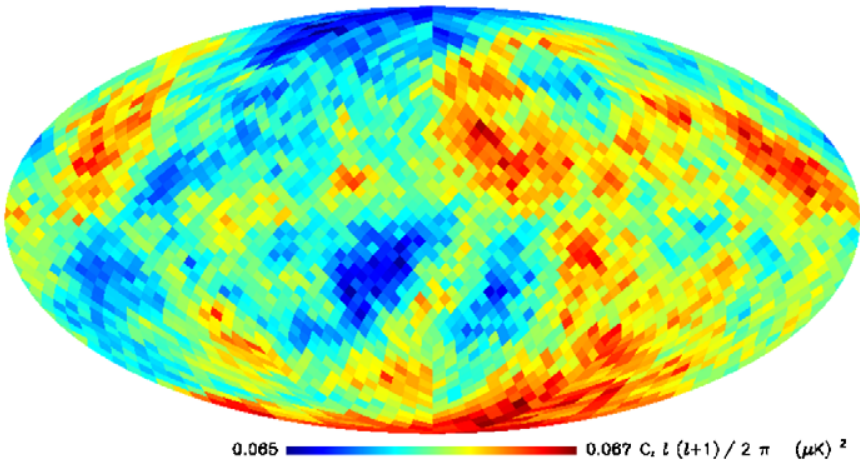

$1=502-601$

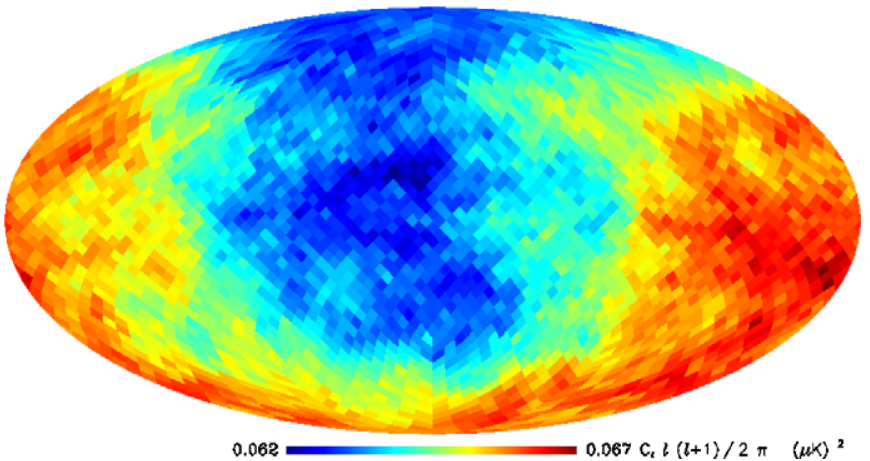

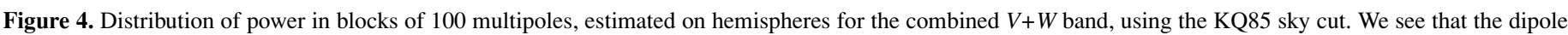
amplitude is stronger in some of the ranges. Note the similarity with the single multipole bins in Figure 6.

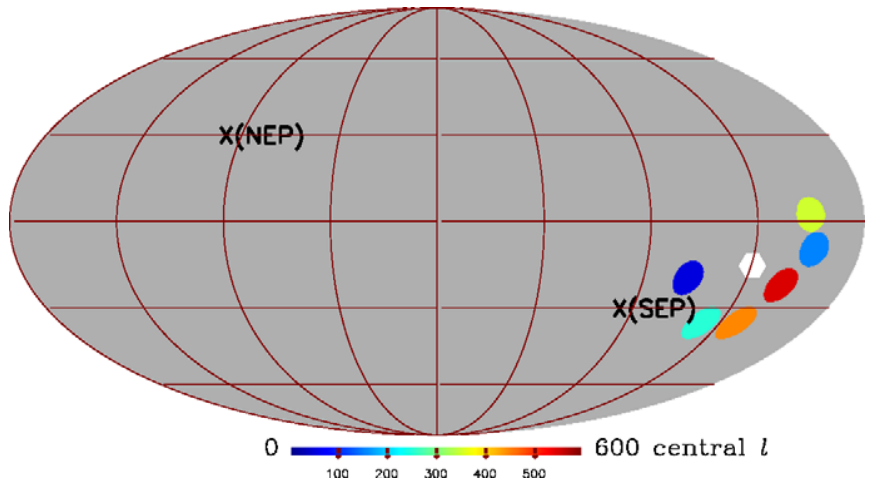

Figure 5. Directions of the dipoles of the power distribution in blocks of 100 multipoles, estimated on disks with diameter 22.5 for the combined $V+W$ band, using the KQ85 sky cut. The color of the disks indicates the center of the given multipole range. The white hexagon indicates the best-fit dipole direction for the full range, $\ell=2-600$. The ecliptic poles are indicated by crosses. obvious in Table 3. Using more localized spectra, the alignment appears even stronger than for hemisphere spectra. Note that for the range $\ell=2-600$, none of the simulations show a similarly strong alignment for any of the disk sizes. In particular, for the 22.5 (diameter) disk results, none of the 9800 simulations have a similarly strong alignment (which is close to a $4 \sigma$ detection of asymmetry). Note also that the alignment is highly significant, also for separate multipole ranges at small and large scales, for instance, $\ell=2-300$ and $\ell=300-600$.

In Figure 7, we show the spectra in the best-fit dipole direction for $\ell=2-600$ for various disk sizes from hemispheres to $45^{\circ}$ (diameter) disks. Also in these plots, we see that the difference between the spectra in the opposite directions becomes larger with more localized spectra. In particular, the first part of the spectrum $\ell=2-100$, as well as the amplitudes of the first two peaks, is clearly different. We have investigated whether also the positions, and not only the amplitudes, of the first two peaks 
$1=2-3$

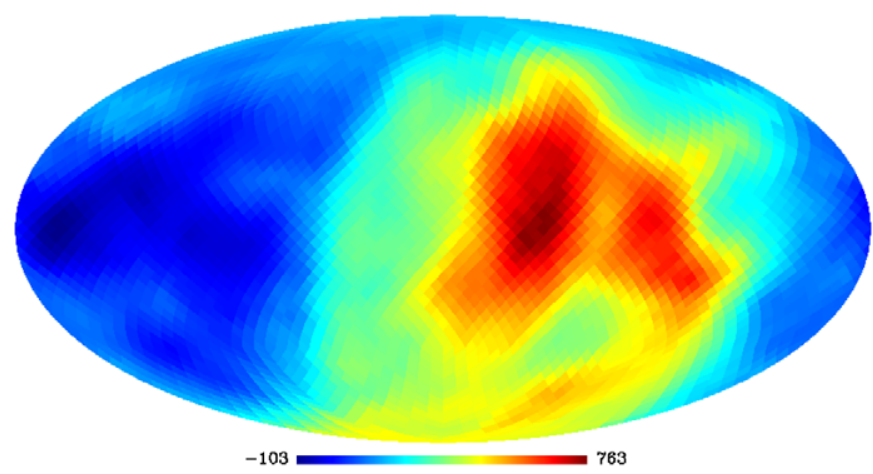

$1=40-41$

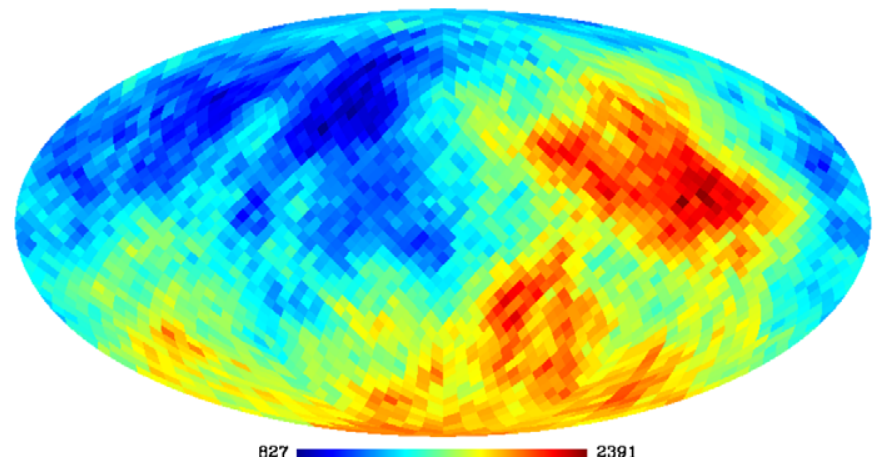

$1=22-23$

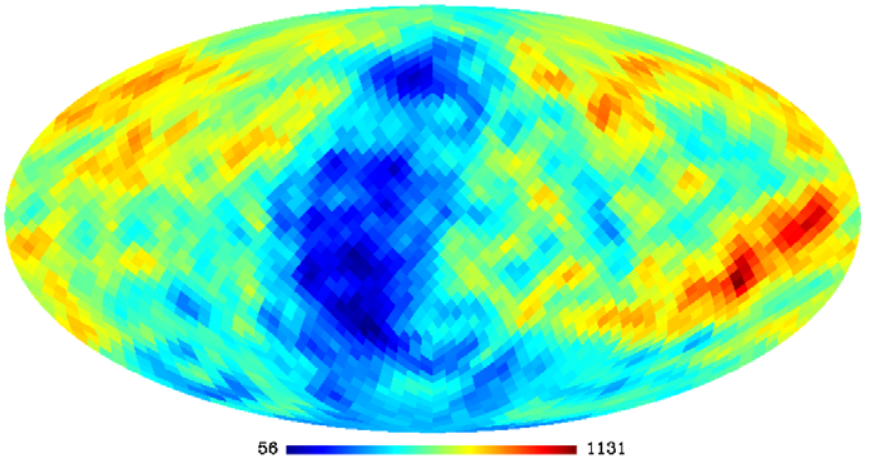

$1=28-29$

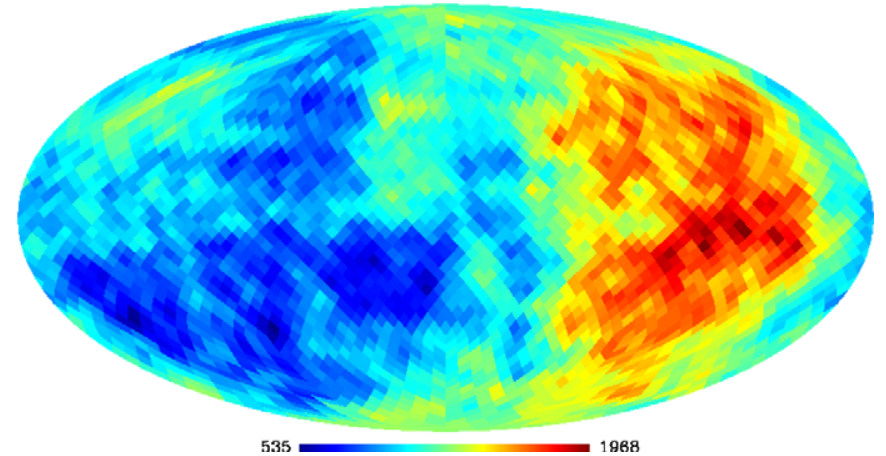

Figure 6. Distribution of power, $\ell(\ell+1) C_{\ell}$, for the multipole ranges $\ell=2-3$ (upper left), $\ell=22-23$ (upper right), $\ell=40-41$ (lower left), and $\ell=28-29$ (lower right).

Table 5

Calculated Mean Angle $\bar{\theta}$ Between the Dipole Directions for all Blocks of 100 Multipoles Within the Given Multipole Range

\begin{tabular}{lccccc}
\hline \hline Disk Size & $180^{\circ}$ & $90^{\circ}$ & $45^{\circ}$ & 22.5 & $90^{\circ}(\mathrm{KQ} 85 \mathrm{~N})$ \\
\hline$\ell=2-800$ & & 6.1 & 8.3 & 9.2 & 53 \\
$\ell=2-700$ & & 0.5 & 0.5 & 0.4 & 15 \\
$\ell=2-600$ & & 0 & 0 & 0 & 1.1 \\
$\ell=2-500$ & 0.1 & 0 & 0 & 0.04 & 3.9 \\
$\ell=2-400$ & 0.1 & 0 & 0.1 & 0.3 & 4.6 \\
$\ell=2-300$ & 0.5 & 0.6 & 0.7 & 1.8 & 11 \\
$\ell=2-200$ & 14 & 13 & 9.2 & 18 & 41 \\
$\ell=200-600$ & & 0 & 0 & 0 & 2.7 \\
$\ell=300-600$ & & 0.1 & 0.1 & 0.1 & 7.3 \\
$\ell=400-600$ & & 3.6 & 0.4 & 0.5 & 6.6 \\
\hline
\end{tabular}

Notes. The numbers given in the table are the percentages of simulated maps with a lower mean angle $\bar{\theta}$. The results are based on 1400 simulations, except for the results for 22.5 disks, which is based on 9800 simulations. Zero entries mean that none of the simulated maps had a similarly low mean angle. The combined $V+W$ map was used for obtaining all results in this table. The KQ85 mask was used, when another mask is not specified. Refer to the text for details of how $\bar{\theta}$ is calculated.

may have a similarly asymmetric distribution on the sky. For the localized spectra obtained on $90^{\circ}$ (diameter) disks, using the $V+W$ map with the KQ85 cut, we made a fit to the first two peaks. From this we constructed a map, $M_{i}$, for each peak with the peak position as a function of $(\theta, \phi)$. No dipole structure similar to what was found for the power spectrum amplitude is seen in these maps.

\subsection{Testing Foregrounds and Systematics}

In this section, we will perform several tests in order to investigate whether foreground residuals or instrumental systematic effects may cause the observed asymmetry. In particular, we will look at the cross-spectra instead of the auto-spectra, we will look at the WMAP data year by year, and finally we will study in detail whether there are still clear signs of the asymmetry outside the $|b|>30$ cut.

The cross-spectra based on $a_{\ell m}$ obtained from different channels and years of observations are less prone to systematic errors, and in particular to uncertainties in the noise model (Hinshaw et al. 2003). We have obtained the hemisphere spectra based on the spectrum obtained as a mean of all 780 possible combinations of the channels $\mathrm{Q}, \mathrm{V}$, and $\mathrm{W}$, as well as the five years of observation. Making simulations with all 780 crossspectra turned out to require too much CPU time, and we were therefore not able to perform a full statistical test using the crossspectra. Using the WMAP data we found that the direction of the dipole, using the maps $M_{i}^{b}$ based on cross-spectra, is consistent with the dipole based on the auto-spectrum.

Similarly, we have studied the direction of the dipole for each single year of observation. We found that these directions are consistent with the directions obtained with the co-added maps with all years included. There is thus no sign of systematic errors in specific years causing the asymmetry. We have also considered the difference between the power distribution maps, $M_{i}(b 1, b 2)$, obtained with different channels. Foreground residuals causing the asymmetry would show up in these differences between channels. The dipole directions of the two difference maps Q-V and V-W are not similar to the direction of asymmetry. There is thus no sign of a frequency-dependent foreground with the dipolar power distribution that we have detected in the individual bands. We further ran simulations to which we added foreground templates at the level expected to be found in the WMAP data. Although the dipole amplitudes were stronger in these simulations, no preferred direction was identified. We 

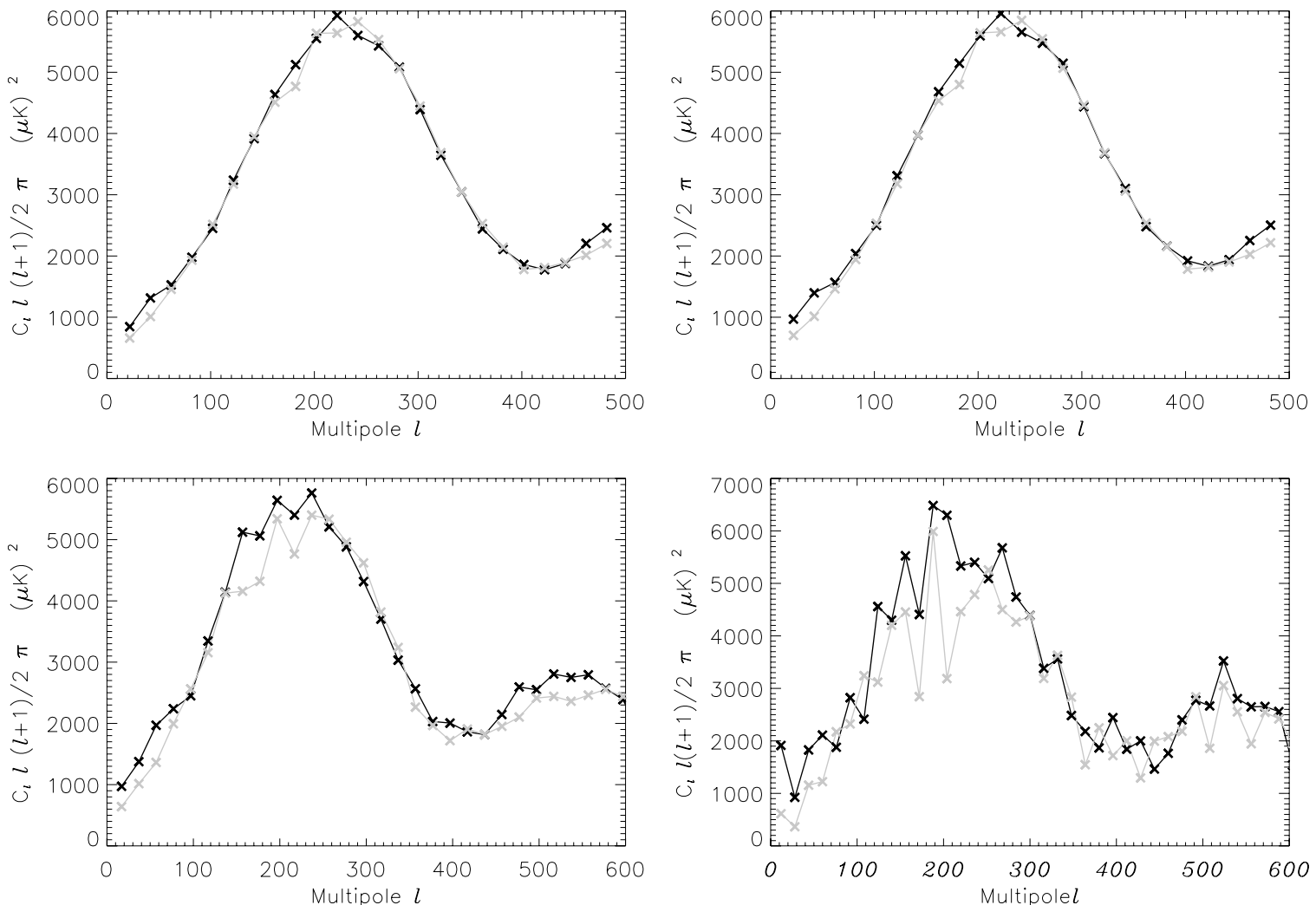

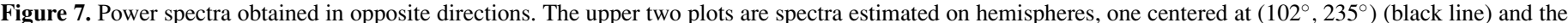

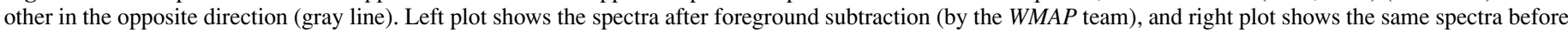

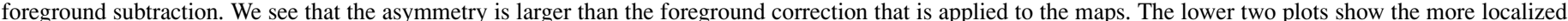

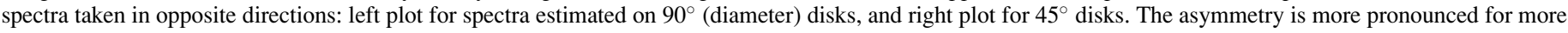
localized spectra.

further checked whether there are more strong outliers in the 22.5 disk spectra near the galactic plane than close to the poles, but the outliers were found to be distributed isotropically on the sphere.

As discussed above, the asymmetry is no longer significant for the $|b|>30$ cut, but the fact that the direction of asymmetry is still (within the error bars) consistent with the direction of asymmetry found for smaller galactic cuts is a strong argument against foreground residuals causing the asymmetry. Still, in order to make sure that the drop in significance for the $60^{\circ}$ cut is not due to the fact that the large mask is excluding some galactic residual causing the asymmetry, we made some further tests. First we made a mask that was equal to the regular KQ85 cut for the southern galactic hemisphere, but had an extended $30^{\circ}$ galactic cut in the northern galactic hemisphere (this is the KQ85N mask). Then we made a similar mask extending only in the southern hemisphere (this is the KQ85S mask).

Using the southern KQ85S mask (for $\ell=2-300$ ), the significance of the asymmetry is still high $(p=1.2 \%)$ for the range $\ell=2-261$ (the direction of asymmetry is consistent with the results above). For the northern KQ85N mask, the significance has dropped to $12 \%$, but the direction of asymmetry is still consistent. Using the more localized spectra estimated on $90^{\circ}$ disks, we see from Table 5 that the alignment between 100 multipole blocks is significant at the $1.1 \%$ level for $\ell=2-600$, using the KQ85N cut. For $\ell=2-400$, the alignment is still significant at the $2 \sigma$ level. This result combined with the fact that the direction of asymmetry has changed little with the large $|b|>30$ cut, as well as the consistency of results using different frequency bands, shows that an explanation of the asymmetry in terms of foreground residuals is difficult to find consistent with the results presented in this paper.

\subsection{Non-flat Amplitude}

We have so far discussed a model where the common dipole has the same amplitude $A_{0}$ over the full multipole range in the best-fit model. We have also tested a model with $A$ decreasing linearly with multipole, as well as a Gaussian shape of the amplitude. Both of these models are described in detail in Section 3.2. In Table 6, we present the results. The first part of the table shows the result with the linear fit, where $\alpha$ is the parameter describing how fast $A$ decreases with multipole. We see that a model with decreasing amplitude is preferred by the data, in fact $\alpha=0$ is excluded at the $3 \sigma$ level. In Figure 8, we show the multipole dependence of the asymmetry. The asymmetry decreases and vanishes close to $\ell=600$, consistent with the results in the previous sections.

In the lower part of the table, the results with the Gaussian model are shown. We show the results for the four-parameter model, where the peak of the Gaussian is forced to $\ell_{\text {peak }}=2$, with the width $\sigma$ allowed to vary, as well as for the fiveparameter model with $\ell_{\text {peak }}$ as an additional free parameter. With the exception of the $45^{\circ}$ disk results, the five-parameter model finds the same best-fit model with $\ell_{\text {peak }}=2$ as the four-parameter model. In Figure 8, we have plotted the bestfit Gaussian model for $90^{\circ}$ disks on top of the linear model. We see that the two models show a consistent decrease in the amplitude of the asymmetry. We conclude that the asymmetry is larger for smaller multipoles and decreasing continuously toward $\ell=600$, where it disappears. 
Table 6

Significances and Directions of the Common Dipole Component for Asymmetric Models with Linearly Decreasing or Gaussian Amplitude Profile

\begin{tabular}{lcrcccc}
\hline \multicolumn{1}{c}{ Mask } & $\alpha\left(10^{-4}\right) / \ell_{\text {peak }}$ & $\theta(\mathrm{deg})$ & $\phi(\mathrm{deg})$ & $\sigma$ & $A_{0}\left(\mu K^{2} / 2 \pi\right)$ & $p(\%)$ \\
\hline \multicolumn{7}{c}{ Linear } \\
KQ85 & $18 \pm 5$ & $99 \pm 21$ & $229 \pm 19$ & & $150 \pm 53$ & 0.6 \\
KQ75 & $18 \pm 8$ & $100 \pm 33$ & $218 \pm 27$ & & $111 \pm 53$ & 17 \\
KQ85 (90 disks) & $15 \pm 5$ & $100 \pm 16$ & $230 \pm 18$ & & $236 \pm 72$ & 1.3 \\
KQ85 (45 ${ }^{\circ}$ disks) & $14 \pm 4$ & $99 \pm 13$ & $228 \pm 16$ & & \\
\hline \multicolumn{7}{c}{ Gauss } \\
\hline KQ85 & NA & $100 \pm 21$ & $228 \pm 19$ & $230 \pm 37$ & $146 \pm 48$ & 0.4 \\
KQ85 & NA & $100 \pm 21$ & $228 \pm 19$ & $230 \pm 37$ & $146 \pm 48$ & 1.7 \\
KQ85 (90 disks) & NA & $100 \pm 16$ & $230 \pm 18$ & $252 \pm 37$ & $239 \pm 74$ & 0.8 \\
KQ85 (90 disks) & $2 \pm 49$ & $100 \pm 16$ & $230 \pm 18$ & $252 \pm 37$ & $239 \pm 74$ & 14 \\
KQ85 (45 disks) & NA & $100 \pm 13$ & $228 \pm 16$ & $312 \pm 42$ & $256 \pm 72$ & 1.4 \\
KQ85 (45 disks) & $408 \pm 55$ & $100 \pm 13$ & $228 \pm 16$ & $312 \pm 41$ & $262 \pm 73$ & 9.1 \\
\hline
\end{tabular}

Notes. We show the significances (in \%), and parameters $(\theta, \phi)$, as well as $\alpha$, for the linear model. For the Gaussian model, we show the best-fit parameters $\sigma$ (included only in the five-parameter fit), and $\ell_{\text {peak }}$. Please refer to the text for details about the asymmetric models and their parameters. The significances specify the percentage of simulated maps with a larger drop in $\chi^{2}$ for the asymmetric model than found in the WMAP data. The results are based on 1400 simulations for the $V+W$ channel.

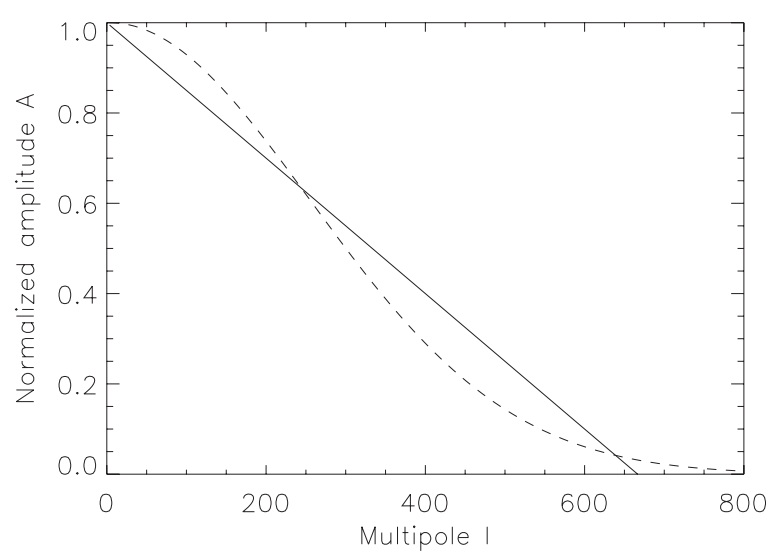

Figure 8. Multipole dependence of the amplitude of asymmetry $A$. We show the results of the fit to a linear and to a Gaussian model of $A$. The results were obtained from spectra estimated on $90^{\circ}$ (diameter) disks, using the $V+W$ band with the KQ85 sky cut.

\section{CONCLUSIONS}

We have reassessed the asymmetry in the distribution of the CMB fluctuation power on the sky reported by Eriksen et al. (2004b) and Hansen et al. (2004b). In order to test whether an anisotropic model of the CMB fluctuations is actually preferred over an isotropic model, taking into account the additional parameters required, we implemented a new model selection procedure. We modeled the asymmetric distribution of power on the sky as a dipole in the power distribution. Note that this is not to say that the CMB fluctuation field has a dipole, rather that the power for a certain scale (multipole) has a dipolar distribution on the sky. We used a model where there is a common dipole component in the power distribution for a set of multipoles in a range $\left[\ell_{\min }, \ell_{\max }\right]$, where $\ell_{\min }$ and $\ell_{\max }$, as well as the direction $(\theta, \phi)$ and the amplitude of the dipole $A_{0}$, were free parameters. We used a $\chi^{2}$ approach to find the best-fit model parameters among these five parameters.

We first investigated a model where we assumed the asymmetry to start at $\ell_{\min }=2$, reducing the number of free parameters in the model to 4 . Using power spectra estimated on hemispheres in the combined $V+W$ band with the KQ85 galactic cut, we found a strong asymmetry in the multipole range $\ell=2-221$, with an axis pointing in the direction $\left(\theta=107^{\circ} \pm 10^{\circ}, \phi=226^{\circ} \pm 10^{\circ}\right)$ (which is the direction where the power is largest). Only $0.3 \%$ of the simulated isotropic maps showed a similarly strong asymmetry. Performing the same test on the $Q, V$, and $W$ bands individually, as well as with different galactic cuts, a similar asymmetry was found. The significance was reduced for larger cuts, but for an extended KQ75 cut (excluding 37\% of the sky), only $1.5 \%$ of the simulated isotropic maps showed a similarly strong common dipole component in the range $\ell=2-300$. Using more localized power spectra estimated on smaller disks, we performed the same tests on the $V+W$ band, using the KQ85 cut. Smaller disks allow faster spherical harmonic transforms and allow the analysis to include multipoles up to $\ell=800$. We found that the range $\ell=2-600$ is asymmetric with the dipole direction $\left(\theta=107^{\circ} \pm 11^{\circ}, \phi=216^{\circ} \pm 10^{\circ}\right)$, using the smallest 22.5 diameter disks. Only $0.4 \%$ of the simulated maps showed similar asymmetry. In Figure 7, we showed the spectra in the two opposite parts of the sky. The spectra are clearly different for the largest scales, as well as around the first two peaks.

Including $\ell_{\min }$ as a free parameter, the same anisotropic model was favored, and the best-fit value for the first multipole with a common dipole was $\ell_{\min }=2$. We therefore concluded that the four-parameter model was sufficient to describe the asymmetry. Testing models with a multipole-dependent dipole amplitude, we found that a model where the asymmetry is maximum for small $\ell$, and decreasing with increasing $\ell$, vanishing at about $\ell \sim 650$, gave a good fit to the data $(0.4 \%)$.

To check whether the asymmetry is present in the full range $\ell=2-600$, or only for some multipoles, we performed a second, simpler, test of asymmetry. We found the dipole direction for the power distribution in multipole ranges of 100 multipoles, $\ell=2-101, \ell=102-201$, etc., to $\ell=502-601$. We thus obtained six dipole directions from six independent multipole ranges. The power distribution in these ranges is shown in Figure 4. We found that these six dipoles were much more aligned in the WMAP data than in isotropic simulations. In fact, using power spectra estimated on 22.5 diameter disks from the combined $V+W$ bands with the KQ85 galactic cut, we found that none of our 9800 simulations showed a similarly strong alignment between these six dipoles. The dipole directions for 
these six ranges are shown in Figure 5. We found that the spatial distribution of CMB fluctuations is strongly correlated between small and large angular scales. Our findings have now also been confirmed by a pixel-based method for $\ell<64$ by Hoftuft et al. (2009).

The fact that all the frequency channels, all years of observations, and also tests using cross power spectra, show a similarly asymmetric distribution, strongly disfavors an explanation in terms of systematic effects and residual galactic foregrounds. Further, in the 5 year WMAP data a different approach to foreground subtraction than for the first year data was applied, still the asymmetry remained significant at the same level for the large scale asymmetry $\ell=2-40$. Hansen et al. (2006) also showed that a blind approach to foreground subtraction did not change this result for large scales.

Our results indicate that the reported common asymmetric axis, extending over a large range in scales, is highly unlikely to be a statistical fluke. Foregrounds and systematic effects do not seem to be probable explanations. The CMB does seem to have an uneven power distribution on the sky over a large range of angular scales. An important task for further research is to find a physical explanation for this asymmetry that can predict possible effects on $\mathrm{CMB}$ polarization to be tested in future experiments.

We acknowledge the use of the HEALPix (Górski et al. 2005) package. F.K.H. is thankful for an OYI grant from the Research Council of Norway. We acknowledge the use of the NOTUR super computing facilities. We acknowledge the use of the Legacy Archive for Microwave Background Data Analysis (LAMBDA). Support for LAMBDA is provided by the NASA Office of Space Science.

\section{REFERENCES}

Ackerman, L., Carroll, S. M., \& Wise, M. B. 2007, Phys. Rev. D, 75, 083502 Bennett, C. L., et al. 2003, ApJS, 148, 1

Boehmer, C. G., \& Mota, D. F. 2008, Phys. Lett., B663, 168

Bridges, M., McEwen, J. D., Lasenby, A. N., \& Hobson, M. P 2007, MNRAS, 377,1473

Cruz, M., Cayon, L., Martinez-Gonzalez, E., Vielva, P., \& Jin, J. 2007, ApJ, 655,11

de Oliveira-Costa, A., Tegmark, M., Zaldarriaga, M., \& Hamilton, A. 2004, Phys. Rev. D, 69, 063516

Erickcek, A. L., Kamionkowski, M., \& Carroll, S. M. A 2008, Phys. Rev. D, 78,123520

Eriksen, H. K., Banday, A. J., Górski, K. M., Hansen, F. K., \& Lilje, P. B. 2007, ApJ, 660, L81
Eriksen, H. K., Banday, A. J., Górski, K. M., \& Lilje, P. B. 2004a, ApJ, 612, 633

Eriksen, H. K., Hansen, F. K., Banday, A. J., Górski, K. M., \& Lilje, P. B. 2004b, ApJ, 605, 14

Eriksen, H. K., Novikov, D. I., Lilje, P. B., Banday, A. J., \& Gorski, K. M. 2004c, ApJ, 612, 64

Gordon, C., Hu, W., Huterer, D., \& Crawford, T. 2005, Phys. Rev. D, 72, 103002

Górski, K. M., Hivon, E., Banday, A. J., Wandelt, B. D., Hansen, F. K., Reinecke, M., \& Bartelman, M. 2005, ApJ, 622, 759

Groeneboom, N. E., \& Eriksen, H. K. 2009, ApJ, 690, 1807

Gumrukcuoglu, A. E., Contaldi, C. R., \& Peloso, M. 2007a, in Proc. 11th Marcel Grossmann Meeting on General Relativity, ed. H. Kleinert, R. T. Jantzen, \& R. Ruffini (Singapore: World Scientific)

Gumrukcuoglu, A. E., Contaldi, C. R., \& Peloso, M. 2007b, J. Cosmol Astropart. Phys. JCAP11(2007)005

Hansen, F. K., Balbi, A., Banday, A. J., \& Gorski, K. M. 2004a, MNRAS, 354, 905

Hansen, F. K., Banday, A. J., Eriksen, H. K., Gorski, K. M., \& Lilje, P. B. 2006, ApJ, 648, 784

Hansen, F. K., Banday, A. J., \& Górski, K. M. 2004b, MNRAS, 354, 641

Hansen, F. K., Cabella, P., Marinucci, D., \& Vittorio, N. 2004c, ApJ, 607, L67

Hansen, F. K., \& Górski, K. M. 2003, MNRAS, 343, 559

Hansen, F. K., Górski, K. M., \& Hivon, E. 2002, MNRAS, 336, 1304

Himmetoglu, B., Contaldi, C. R., \& Peloso, M. 2009, Phys. Rev. Lett., 102, 111301

Hinshaw, G., et al. 2003, ApJS, 148, 135

Hinshaw, G., et al. 2007, ApJS, 170, 288

Hinshaw, G., et al. 2009, ApJS, 180, 225

Hivon, E., Górski, K. M., Netterfield, C. B., Crill, B. P., Prunet, S., \& Hansen, F. K. 2002, ApJ, 567, 2

Hoftuft, J., Eriksen, H. K., Banday, A. J., Gorski, K. M., Hansen, F. K., \& Lilje, P. B. 2009, ApJ, 699, 985

Jaffe, T. R., Banday, A. J., Eriksen, H. K., Gorski, K. M., \& Hansen, F. K 2005, ApJ, 629, L1

Jaffe, T. R., Banday, A. J., Eriksen, H. K., Gorski, K. M., \& Hansen, F. K. 2006a, A\&A, 460, 393

Jaffe, T. R., Hervik, S., Banday, A. J., \& Gorski, K. M. 2006b, ApJ, 644, 701

Koivisto, T. S., \& Mota, D. F. 2008a, J. Cosmol. Astropart. Phys. JCAP08(2008)021

Koivisto, T. S., \& Mota, D. F. 2008b, ApJ, 679, 1

Land, K., \& Magueijo, J. 2005a, Phys. Rev. Lett., 95, 071301

Land, K., \& Magueijo, J. 2005b, MNRAS, 357, 994

Land, K., \& Magueijo, J. 2007, MNRAS, 378, 153

Larson, D. L., \& Wandelt, B. D. 2004, ApJ, 613, L85

McEwen, J. D., Hobson, M. P., Lasenby, A. N., \& Mortlock, D. J. 2005, MNRAS, 359,1583

McEwen, J. D., Hobson, M. P., Lasenby, A. N., \& Mortlock, D. J. 2006, MNRAS, 371, L50

Park, C.-G. 2004, MNRAS, 349, 313

Pietrobon, D., et al. 2008, Phys. Rev. D, 78, 103504

Spergel, D. N., et al. 2007, ApJS, 170, 377

Tegmark, M., de Oliveira-Costa, A., \& Hamilton, A. J. 2003, Phys. Rev. D, 68 , 123523

Vielva, P., Martinez-Gonzalez, E., Barreiro, R. B., Sanz, J. L., \& Cayon, L. 2004, ApJ, 609, 22 